\title{
RAZOR trial: analysis of 3-year follow-up: an era of robotic radical cystectomy: is it a new beginning?
}

\author{
Satish Kumar Ranjan* (1)
}

\begin{abstract}
RAZOR (Randomized Open versus Robotic Cystectomy) trial is a phase 3 randomized control trial comparing robotassisted laparoscopic radical cystectomy with open radical cystectomy for the treatment of bladder cancer published by Parekh et.al. Three-year follow-up analysis of this trial showed that there was no difference in overall survival, progression-free survival, and local or distant recurrence in both the group. The present report will discuss this landmark trial in brief in the context of the advantage and feasibility of robotic radical cystectomy in the developing world.
\end{abstract}

\section{Summary}

RAZOR [1] (Randomized Open versus Robotic Cystectomy) trial is a multicentre (done at 15 medical centers), randomized, open-label, phase 3, non-inferiority trial comparing robot-assisted laparoscopic radical cystectomy(RARC) with open radical cystectomy(ORC) for the treatment of bladder cancer (CaUB). Patients aged 18 years or older and who had biopsy-proven non-metastatic CaUB (T1-T4, N0-N1) or refractory carcinoma in situ (CIS) were included for study and who had previous history of open abdominal-pelvic surgery, any contraindications to initiate or maintain pneumoperitoneum and pregnant women were excluded. Patients were randomized to 1:1 in both the group via a web-based system.

Between 1st July 2011 to 18th November 2014, a total of 350 patients were randomly assigned to RARP (176) and ORC (174) treatment group. After the exclusion of patients who received treatment other than the initial allotted treatment 150 patients in RARC and 152 patients in the ORC, group were included for per-protocol (PP) and 159 and 153 in RARC and ORC group respectively for modified intention to treat analysis (ITT). Further, it was stratified by type of urinary diversion (incontinent

*Correspondence: docsatis48@gmail.com

Department of Urology, All India Institute of Medical Sciences, Rishikesh 249203, Uttarakhand, India or continent), clinical T stage (carcinoma in situ, T1-T2, or T3-T4), and Eastern Cooperative Oncology Group (ECOG) performance status $(0-1$, or $\geq 2)$.

In the RARC group, 27\% (41) and ORC group 36\% (55) of patients received neoadjuvant chemotherapy (NAC). All the urinary diversions were extracorporeal only according to surgeons and patient preferences. Neobladder and ileal conduit were made in $24 \%$ ( 36 ) and $75 \%$ (113) and 20\% (30) and 80\% (122) of patients in the RARP and ORC group respectively. Only 1 patient had continent cutaneous diversion (CCD) in the RARP group and none in ORC.

All the cystectomies were done by experienced surgeons who had dedicated training in the management of bladder cancer and performed at least ten radical cystectomy in the previous year. Pelvic lymph node dissection was done according to institutional protocol (standard or extended). Cost analysis was not done, of course, robotic surgery carries a high cost.

The first report of the RAZOR trial, a study of 2-year follow-up was published in June 2018 [1]. The primary endpoint was progression-free survival (PFS) and the study showed that no significant difference in PFS in both the arm, i.e., non-inferiority of RARC over ORC. Two-year PFS was $72.3 \%$ (95\% CI 64.3 to 78.8 ) in the robotic cystectomy group and $71.6 \%$ (95\% CI 63.6 to 
78.2) in the open cystectomy group (difference 0.7\% [95\% CI 9.6-10.9; $P=0.90 ; P_{\text {non-inferiority }}=0.001$ ) [1].

In secondary endpoints, the estimated mean blood loss was significantly lower $(<0.0001)$ in RARP $(300 \mathrm{ml})$ than ORC $(700 \mathrm{ml})$. Hospital stay of less than 5 days was also lower in RARP $(29 \%)$ than the ORC $(18 \%)$ group of patients $(p=0.0407)$. the operating time was significantly higher in the robotic group ( $428 \mathrm{~min}$ vs. $361 \mathrm{~min})$. No significant differences in complications ( $\leq 90$ days) were noted in both groups. Also, there were no statistically significant differences between the two groups in tumor type, staging, lymph node yield, and positive surgical margin $(p>0.05)$.

The second report of the RAZOR trial, a study of 3-year follow-up was published in March 2020 [2]. In this study, the authors analyzed the per-protocol population of 302 patients (150-RARC, 152-ORC). At 36 months of follow-up, results of PFS and overall survival (OS, $P$-0.432) were comparable in both groups. PFS was $68.4 \%$ (95\% CI 60.1-75.3) in the robotic group and $65.4 \%$ (95\% CI $56.8-72.7)$ in the open group ( $P$ $0.600)$. The HR of OS was $73.9 \%$ (95\% CI 65.5-80.5) in the robotic group and $68.5 \%$ (95\% CI 59.8-75.7) in the open group ( Table 1 ).

There was no significant difference between the 2 groups in local or distant recurrences. Local recurrence was reported as $6(4 \%)$ in RARP and $4(2.6 \%)$ in ORC $(P-0.541)$ and distant in $33(22 \%)$ in the robotic and $35(23 \%)$ in the open cystectomy group $(p-0.605)$. The median time to recurrence was 10.2 months in the robotic group vs 6.3 months in the open group. Higher pathological stage and positive margins were significant predictors of recurrence, PFS, and OS. It was the patient's age greater than 70 years, poor performance status, and major complications, and not the surgical approach which were significant predictors of 36-month progression-free survival.

The authors conclude that there was no difference in OS [2], PFS [1, 2] local, or distant recurrence [2] in the RARC and ORC group in follow-up of 3 years.

Table 1 Comparing oncological outcome after 2-year and 3-year follow-up

\begin{tabular}{lll}
\hline Oncological outcome & 2-year follow up & 3-year follow up \\
\hline Progression free & $72 \cdot 3 \%$ in RARC vs & $68.4 \%$ in RARC vs \\
survival & $71 \cdot 6 \%$ in ORC & $65.4 \%$ in RARC $(p-0.6)$ \\
& $\begin{array}{l}(p-0.9, \\
\left.P_{\text {noninferiority }}-0.001\right)\end{array}$ & \\
Overall survival & NR & $73.9 \%$ in RARC vs \\
& & $68.5 \%$ in ORC $(p-0.334)$ \\
\hline
\end{tabular}

RARC robot-assisted radical cystectomy, ORC Open radical cystectomy, NR not reported

\section{Commentary}

This prospective phase 3 randomized control trial (RCT) [1] provides level 1 evidence of non-inferiority of robotic radical cystectomy (RARC) over open radical cystectomy (ORC). To the best of my knowledge, this is the largest prospective randomized study comparing time to recurrence, PFS, and OS in robotic cystectomy to open cystectomy.

A small prospective randomized single-center noninferiority study by Nix J et al. of 41 patients, showed no significant difference in overall complications, length of hospital stay, pathological stage, and lymph node yield in RARC (21 patients) and ORC (20 patients) group [3]. A study by Stein et al. of 1054 patients, who underwent ORC showed a 5-year OS of $66 \%$ and recurrence-free survival of (RFS) of $68 \%$, comparable to the present study [4].

An early study on robotic surgeries and minimally invasive surgeries reported that increased risk of peritoneal seeding, port-site metastasis, and positive margins but later it is clarified and proved that tumor biology itself, not the surgical modality is responsible for this particular finding. An important limitation of robotic radical cystectomy is the lack of tactile feedback which may increase the possibility of positive margins in locally advanced cases but any such significant finding was not observed in the study.

In developing countries like India where there is no universal coverage of health insurance, the true benefit of robotic surgery (radical cystectomy) is questionable due to a lack of uniform data on outcomes and individual cost [5]. This landmark trial (RAZOR) provides the non-inferiority of RARC over ORC, but a large superiority trial is the need of the hour to provide true benefit.

\section{Abbreviations}

RAZOR: Randomized open versus robotic cystectomy; RARC: Robot-assisted laparoscopic radical cystectomy; ORC: Open radical cystectomy; CaUB: Carcinoma urinary bladder; CIS: Carcinoma in situ; OS: Overall survival; PFS:

Progression-free survival; RFS: Recurrence free survival.

\section{Acknowledgements \\ None.}

\section{Authors' contributions}

The author read and approved the final manuscript.

\section{Funding}

None.

\section{Availability of data and materials}

Data sharing does not apply to this article as no datasets were generated or analyzed during the current study, all data were taken from RAZOR trial study*. Venkatramani V, Reis IM, Castle EP, Gonzalgo ML, Woods ME, Svatek RS, et al. Predictors of Recurrence, and Progression-Free and Overall Survival following Open versus Robotic Radical Cystectomy: Analysis from the RAZOR Trial with a 3-Year Follow-up. J Urol 2020;203:522-9. 


\section{Declarations}

Ethics approval and consent to participate

Not applicable.

\section{Consent for publication}

Not applicable.

\section{Competing interests}

The author declares no competing interests.

Received: 29 April 2020 Accepted: 16 June 2021

Published online: 27 June 2021

\section{References}

1. Parekh DJ, Reis IM, Castle EP, Gonzalgo ML, Woods ME, Svatek RS, Weizer AZ, Konety BR, Tollefson M, Krupski TL, Smith ND (2018) Robot-assisted radical cystectomy versus open radical cystectomy in patients with bladder cancer (RAZOR): an open-label, randomised, phase 3, non-inferiority trial. The Lancet 391(10139):2525-2536
2. Venkatramani V, Reis IM, Castle EP, Gonzalgo ML, Woods ME, Svatek RS et al (2020) Predictors of recurrence, and progression-free and overall survival following open versus robotic radical cystectomy: analysis from the RAZOR trial with a 3-year followup. J Urol 203:522-529

3. Nix J, Smith A, Kurpad R, Nielsen ME, Wallen EM, Pruthi RS (2010) Prospective randomized controlled trial of robotic versus open radical cystectomy for bladder cancer: perioperative and pathologic results. Eur Urol 57:196-201

4. Stein JP, Lieskovsky G, Cote R, Groshen S, Feng AC, Boyd S et al (2001) Radical cystectomy in the treatment of invasive bladder cancer: longterm results in 1,054 patients. J Clin Oncol 19:666-675

5. Bora GS, Narain TA, Sharma AP, Mavuduru RS et al (2020) Robot-assisted surgery in India: A SWOT analysis. Indian J Urol 36:1-3

\section{Publisher's note}

Springer Nature remains neutral with regard to jurisdictional claims in published maps and institutional affiliations.

\section{Submit your manuscript to a SpringerOpen ${ }^{\circ}$ journal and benefit from:}

- Convenient online submission

- Rigorous peer review

- Open access: articles freely available online

- High visibility within the field

- Retaining the copyright to your article

Submit your next manuscript at $\boldsymbol{\nabla}$ springeropen.com 